\title{
Universal Gate Set for Continuous-Variable Quantum Computation with Microwave Circuits
}

\author{
Timo Hillmann $\odot,{ }^{1,2}$ Fernando Quijandría $\odot,{ }^{1}$ Göran Johansson, ${ }^{1}$ Alessandro Ferraro, ${ }^{3}$ \\ Simone Gasparinetti, ${ }^{1}$ and Giulia Ferrini ${ }^{1}$ \\ ${ }^{1}$ Department of Microtechnology and Nanoscience (MC2), Chalmers University of Technology, SE-412 96 Gothenburg, Sweden \\ ${ }^{2}$ Institut für Theorie der Statistischen Physik, RWTH Aachen, 52056 Aachen, Germany \\ ${ }^{3}$ Centre for Theoretical Atomic, Molecular and Optical Physics, Queen's University Belfast, Belfast BT7 INN, United Kingdom
}

(Received 7 February 2020; accepted 16 September 2020; published 12 October 2020)

\begin{abstract}
We provide an explicit construction of a universal gate set for continuous-variable quantum computation with microwave circuits. Such a universal set has been first proposed in quantum-optical setups, but its experimental implementation has remained elusive in that domain due to the difficulties in engineering strong nonlinearities. Here, we show that a realistic three-wave mixing microwave architecture based on the superconducting nonlinear asymmetric inductive element [Frattini et al., Appl. Phys. Lett. 110, 222603 (2017)] allows us to overcome this difficulty. As an application, we show that this architecture allows for the generation of a cubic phase state with an experimentally feasible procedure. This work highlights a practical advantage of microwave circuits with respect to optical systems for the purpose of engineering non-Gaussian states and opens the quest for continuous-variable algorithms based on few repetitions of elementary gates from the continuous-variable universal set.
\end{abstract}

DOI: 10.1103/PhysRevLett.125.160501

Introduction.-The ability to control and manipulate quantum systems has reached an unprecedented level in the past decades $[1,2]$. Quantum computation stems as one of the most promising potential applications of this enhanced controllability of quantum systems [3-5]. As an alternative to the use of two-level systems for quantum information encoding, continuous-variable (CV) architectures have emerged, where the underlying hardware consists in quantized radiation, either with optical devices [1] or microwaves [6] or in cavity optomechanics [7].

The theoretical setting for quantum computation with $\mathrm{CV}$-based architectures has been laid down in a seminal paper by Lloyd and Braunstein [8]. There, universal quantum computation in CVs is defined as the ability of implementing any evolution corresponding to Hamiltonians that are arbitrary polynomials in the mode quadratures. The basic ingredients to achieve CV universality are a set of Gaussian gates and a single non-Gaussian gate, which can be chosen arbitrarily among the polynomials of degree higher than 2 in the quadratures of the quantized modes. The ability to perform arbitrary sequences of these elementary quantum gates ensures universal quantum computation [8].

Published by the American Physical Society under the terms of the Creative Commons Attribution 4.0 International license. Further distribution of this work must maintain attribution to the author(s) and the published article's title, journal citation, and DOI. Funded by Bibsam.
Since then, the community of quantum opticians has devoted considerable theoretical as well as experimental effort toward developing the building blocks for $\mathrm{CV}$ universality. In this framework, the experimental challenge consists in achieving a non-Gaussian operation. Experimental effort has focused on photon subtraction [9-16], photon detection [17-19], and the use of ancillary low photon-number states combined with homodyne detection [20,21] as ways to achieve probabilistic nonGaussian transformations, resulting, however, in low success probabilities [22] and limited versatility. In particular, much of the effort has been dedicated to engineer the so-called "cubic phase gate" or, alternatively, to generate a "cubic phase state" [23]. Availability of the latter state allows for engineering a cubic phase gate by gate teleportation $[15,16,22-26]$ and thereby promotes the set of Gaussian operations to a universal set $[8,27]$. Having at one's disposal such a cubic gate would allow one, in particular, to engineer Gottesman-Kitaev-Preskill (GKP) states [23,28], which have been shown to yield fault tolerance [23,29-32]. Despite these efforts, the generation of a cubic phase state, as well as the implementation of a cubic phase gate, have remained elusive, due to the weakness of the nonlinearities that are available in the optical regime. Alternatively, deterministic nonlinear gates in strongly coupled quantum electrodynamics (QED) setups [33] as well as the dissipative stabilization of cubic phase states in optomechanical systems have been proposed $[34,35]$. 
In microwave quantum optics, commonly referred to as circuit QED (cQED), nonlinear photon interactions are made possible via the use of Josephson-junction-based devices [36]. Through a high degree of control in their interactions with linear resonators, they led to prominent realizations such as the generation of arbitrary Fock states and their superpositions [37], Schrödinger cat states [38,39], and Gottesman-Kitaev-Preskill states [40].

In particular, the superconducting nonlinear asymmetric inductive element (SNAIL) [41-43] enables the mediation of degenerate and nondegenerate three-photon interactions without spurious effects commonplace in CQED through a combination of its geometry and control through external magnetic fields.

The CV notion of universality has not been studied thoroughly in these systems yet, nor has it ever been achieved experimentally. Here, we bridge between the optical and microwave approaches and show explicitly that a parametrically modulated microwave architecture making use of a SNAIL allows for implementing a universal gate set for $\mathrm{CV}$ quantum computation, in the sense of the CV universality notion recalled above [8]. As an application, we show that a state-of-the-art microwave platform allows for the generation of a cubic phase state-a long sought-after aspiration for the quantum optics community.

Universal gate set in continuous-variables. - A universal gate set for $\mathrm{CV}$ quantum computation is provided by the following operations [8]:

$\left\{e^{i \hat{q}_{k} s_{1}}, e^{i\left(\hat{q}_{k} \hat{p}_{k}+\hat{p}_{k} \hat{q}_{k}\right) s_{2}}, e^{i\left(\hat{p}_{k} \hat{q}_{l}-\hat{q}_{k} \hat{p}_{l}\right)}, e^{i(\pi / 4)\left(\hat{q}_{k}^{2}+\hat{p}_{k}^{2}\right)}, e^{i \hat{q}_{k}^{3} \gamma}\right\}$,

where $\hat{q}_{k}=\left(\hat{a}_{k}+\hat{a}_{k}^{\dagger}\right) / \sqrt{2}$ and $\hat{p}_{k}=\left(\hat{a}_{k}-\hat{a}_{k}^{\dagger}\right) /(i \sqrt{2})$ are the quadrature operators for mode $k$ satisfying the canonical commutation relation $\left[\hat{q}_{k}, \hat{p}_{l}\right]=i \delta_{k l}$ (from here on, $\hbar=1$ and we drop the mode index if only a single mode is relevant).

The operations in Eq. (1) excluding $e^{i \hat{q}^{3} \gamma}$ represent, respectively, the displacement, squeezing, beam splitter, and Fourier-transform operators (where $s_{i} \in \mathbb{R}$ for all $i$ ) and are universal for Gaussian operations; i.e., they allow implementing any arbitrary quadratic Hamiltonian. Addition of the cubic phase gate $e^{i \hat{q}^{3} \gamma}$, where one value of the cubicity $\gamma$ is sufficient [8,26,28,44-46], allows promoting the Gaussian set of operations to universal quantum computation. Indeed, following Ref. [8], if we can apply the Hamiltonians $\hat{A}$ and $\hat{B}$ for a time $\delta t$, then we can approximate the evolution under their commutator for a time $\delta t^{2}$ by means of the relation

$$
e^{-[\hat{A}, \hat{B}] \delta t^{2}}=e^{i \hat{A} \delta t} e^{i \hat{B} \delta t} e^{-i \hat{A} \delta t} e^{-i \hat{B} \delta t}+\mathcal{O}\left(\delta t^{3}\right) .
$$

Commuting a polynomial in $\hat{q}$ and $\hat{p}$ with $\hat{q}$ and $\hat{p}$ themselves reduces the order of the polynomial by at least 1. Commuting with quadratic Hamiltonians never increases the order. Finally, commuting with a polynomial of order 3 or higher increases the order by at least 1 . Hence, one can achieve arbitrary Hermitian polynomials of any order in $\hat{q}$ and $\hat{p}$ by commuting properly the Gaussian operations with an applied Hamiltonian of order 3 or higher. Consequently, the set in Eq. (1) is universal. Direct application of Eq. (2) may result in a significant number of operations in order to approximate a desired Hamiltonian. More efficient schemes involving nested operations as well as numerical optimization may provide shorter gate sequences for achieving the same approximate Hamiltonian evolution [51-53]. However, the approach described above will suffice for our purpose of establishing a proof of principle for universality with microwave circuits. We are now going to introduce a cQED architecture that is instrumental to implement the universal gate set in Eq. (1).

Microwave circuit for $C V$ universal quantum computation.-Interactions between microwave photons in superconducting circuits can be realized by coupling the modes of interest to Josephson junctions acting as nonlinear, low-loss inductive elements with potential energy $U(\varphi)=E_{J}[1-\cos (\varphi)]$, where $\varphi$ is the superconducting phase across the junction and $E_{J}$ is the Josephson energy $[36,54]$. When Josephson junctions are arranged in a loop configuration, as in a dc superconducting quantum interference device (SQUID), the Josephson potential energy depends on the magnetic flux threading the loop, allowing for in situ static tuning of the potential, as well as its parametric modulation [55,56]. Photon-photon interactions have been demonstrated in resonators terminated by dc SQUIDs by introducing suitable drives to resonantly select specific $n$-photon processes from the Josephson potential. This potential has even parity for both a single junction and a symmetric SQUID, resulting in mixing processes with an even number of photons, such as fourwave mixing [57]. As has been recently demonstrated, an asymmetry between the SQUID junctions introduces an odd contribution to the potential, enabling three-wave mixing (as well as higher-order odd photon interactions) [58,59]. However, the even contribution still results in undesired terms, most notably, self- and cross-Kerr interactions, that contain an equal number of creation and annihilation operators and are consequently resonant (nonrotating) in any reference frame. To overcome this challenge, the SNAIL was recently introduced [41-43] in the context of Kerr-free three-wave mixing and parametric amplification. Here we propose a tunable resonator design based on a SNAIL and show that by a two-tone flux modulation we can resonantly select all processes comprising the cubic interaction $\left(\hat{a}+\hat{a}^{\dagger}\right)^{3}$ as we will detail later. The SNAIL loop consists of $n$ large Josephson junctions in parallel with a single smaller junction with Josephson energies $E_{J}$ and $\alpha E_{J}(\alpha<1)$, respectively [Fig. 1(a)]. By threading an external magnetic flux $\Phi_{\text {ext }}$ 


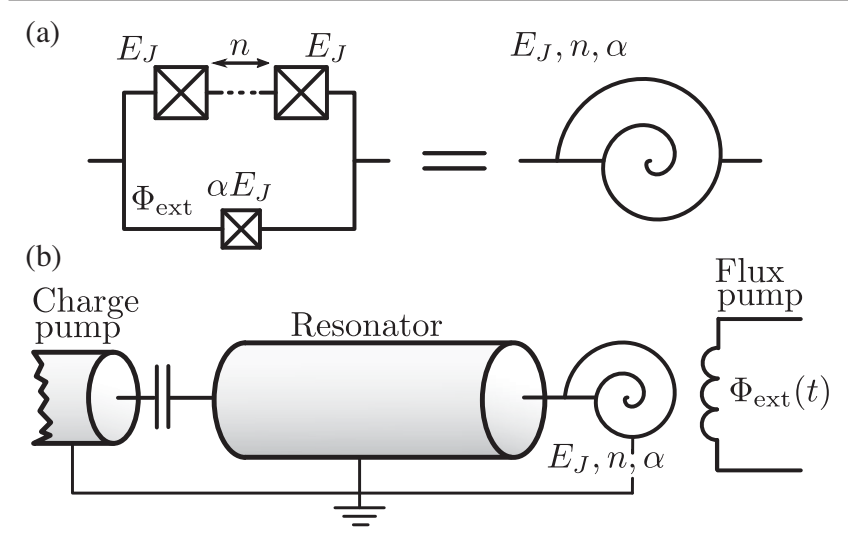

FIG. 1. (a) Circuit representation of the SNAIL composed of $n$ large Josephson junctions of energy $E_{J}$ and a single smaller one of energy $\alpha E_{J}$. Following Ref. [41], we represent this subcircuit by a snail-like symbol. (b) Sketch of our proposed architecture. The quarter wavelength transmission line resonator is terminated into a SNAIL at the right end and capacitively coupled to an input transmission line at the left end through which microwave signals for control can be fed. The SNAIL potential can be tuned and modulated through an external flux line.

through the loop, the inductive energy of the SNAIL circuit can be written as [41]

$$
U_{\mathrm{SNAIL}}(\varphi)=-\alpha E_{J} \cos (\varphi)-n E_{J} \cos \left(\frac{\varphi_{\mathrm{ext}}-\varphi}{n}\right)
$$

where $\varphi$ is the superconducting phase across the small junction, $\varphi_{\mathrm{ext}}=2 \pi \Phi_{\mathrm{ext}} / \Phi_{0}$ is the reduced applied magnetic flux, and $\Phi_{0}$ is the magnetic flux quantum. The advantage of the SNAIL circuit over traditional SQUIDs is that through its design, resulting in specific parameters $n, \alpha$, in addition to the external flux $\Phi_{\text {ext }}$, its potential landscape around a minimum $\varphi_{\min }$ can be tailored. Then we can Taylor expand (3) around this value as $U_{\text {SNAIL }}(\varphi)=U_{\text {SNAIL }}\left(\varphi_{\text {min }}\right)+\sum_{m>1} c_{m}\left(\varphi-\varphi_{\text {min }}\right)^{m} / m !$. The three-wave mixing capability of this device corresponds to setting the coefficient of the fourth-order term identical to zero $\left(c_{4}=0\right)$ [41], so that the leading nonlinear term is the cubic one.

Our proposed architecture is a quarter wavelength transmission line resonator of length $d$ terminated with a SNAIL loop in one end [Fig. 1(b)]. We describe the state of the resonator in terms of the superconducting phase field $\varphi(x, t)$. For our purposes, the phase at the position of the SNAIL $\varphi_{S}=\varphi(d, t)$ is assumed to be small. This means that the current flowing through the Josephson junctions is smaller than their corresponding critical currents. If this holds, the Lagrange equations of motion which determine the normal modes of the resonator-SNAIL system can be linearized, allowing one to obtain the system Hamiltonian. The nonlinear corrections are reintroduced perturbatively [46]. The resonator is also weakly coupled to an input transmission line [Fig. 1(b)], which allows driving the resonator field.

We choose the SNAIL parameters $n, \alpha$, and $\Phi_{\text {ext }}$ in order to operate the device free of Kerr interactions. Furthermore, we propose to endow this device of flux tunability in order to fully exploit the third-order interaction. For this, we apply a periodically modulated reduced external flux of the form

$$
\varphi_{\mathrm{ext}}(t)=\varphi_{\mathrm{ext}}^{\mathrm{dc}}+\varphi_{\mathrm{ext}}^{\mathrm{ac}}(t),
$$

where $\varphi_{\mathrm{ext}}^{\mathrm{dc}}$ is the static part of the flux and $\varphi_{\mathrm{ext}}^{\mathrm{ac}}(t)$ is the time-dependent modulation. The latter satisfies $\left|\varphi_{\mathrm{ext}}^{\mathrm{ac}}(t)\right| \ll 1$. This is required in order to remain near the equilibrium point and to not excite higher nonlinear processes. We consider the SNAIL potential up to second order in $\varphi_{\mathrm{ext}}^{\mathrm{ac}}(t)$. As customary, we follow the canonical quantization recipe for the resonator [46]. Upon quantization, the Hamiltonian describing our tunable resonator is

$$
\begin{aligned}
\hat{H}= & \omega_{r} \hat{a}^{\dagger} \hat{a}+g_{1}(t)\left(\hat{a}+\hat{a}^{\dagger}\right)+g_{2}(t)\left(\hat{a}+\hat{a}^{\dagger}\right)^{2} \\
& +g_{3}(t)\left(\hat{a}+\hat{a}^{\dagger}\right)^{3}+g_{4}(t)\left(\hat{a}+\hat{a}^{\dagger}\right)^{4},
\end{aligned}
$$

with time-dependent coefficients $g_{i}(t), i=1, \ldots, 4$ to be discussed below. Here $\omega_{r}$ is the resonance frequency of the transmission line resonator modified by the presence of the SNAIL. Because of the modulation of the potential around its minimum, we gain linear and quadratic contributions in addition to the cubic potential. Notice that we have also included a quartic contribution. The reason is twofold. First, due to the time-dependent modulation, the condition $c_{4}=0$ is not always satisfied. Second, as demonstrated in Ref. [42], the cubic interaction leads to fourth-order renormalization effects. In this context, the Kerr-free operation point results from the interplay of third- and fourth-order nonlinearities. The time dependence of $g_{1}$ and $g_{3}$ is proportional to $\varphi_{\mathrm{ext}}^{\mathrm{ac}}(t)$ and that of $g_{2}$ and $g_{4}$ to $\varphi_{\mathrm{ext}}^{\mathrm{ac}}(t)^{2}$. The perturbative treatment of the nonlinearity leads to the hierarchy $\omega_{r} \gg\left|g_{i}\right|$.

Engineering of Gaussian gates.-We start our demonstration of CV universality by showing that this architecture is capable of implementing the Gaussian operations in (1). For this, a modulation of the flux is not required, i.e., $\varphi_{\mathrm{ext}}^{\mathrm{ac}}(t)=0$ in Eq. (4). In this case, the Hamiltonian (5) reduces to $\hat{H}=\omega_{r} \hat{a}^{\dagger} \hat{a}+g_{3}^{\mathrm{dc}}\left(\hat{a}+\hat{a}^{\dagger}\right)^{3}+g_{4}^{\mathrm{dc}}\left(\hat{a}+\hat{a}^{\dagger}\right)^{4}$, with the couplings $g_{3}^{\mathrm{dc}}$ and $g_{4}^{\mathrm{dc}}$ depending only on the microscopic parameters of the circuit as well as the static external flux. In order to engineer a squeezing operation, we apply an off-resonant microwave tone of frequency $\omega_{p}=2 \omega_{r}$ through the input transmission line. As discussed in Ref. [43], in a frame rotating at the resonator frequency $\omega_{r}$ the system is described by the effective Hamiltonian $\hat{H}_{\mathrm{sq}}=-(i / 2)\left(\xi \hat{a}^{\dagger 2}-\bar{\xi} \hat{a}^{2}\right)$, where the parameter $\xi$ depends on $g_{3}^{\mathrm{dc}}$ as well as on the amplitude of the drive. In particular, choosing $\xi$ real allows us to obtain the 
squeezing operation in Eq. (1). This is the basis of SNAILbased parametric amplification $[42,43]$. The Fourier transform $e^{i(\pi / 4)\left(\hat{q}^{2}+\hat{p}^{2}\right)}$ follows from the free evolution of the system, i.e., the evolution under the resonator Hamiltonian $\omega_{r} \hat{a}^{\dagger} \hat{a}$ in the absence of any external modulation. As customary, a displacement operation is implemented by means of a microwave tone near resonance with mode $\hat{a}$. Finally, a tunable beam-splitter interaction can be achieved by coupling two resonator-SNAIL units via a parametrically modulated dc SQUID or a tunable gap qubit or mediating a static nonlinear coupling via time-dependent drivings of both resonators [60-62].

Engineering a nonlinear gate.-The power of our proposal relies on the realization of the interaction term $\hat{q}^{3}$ which has been experimentally elusive so far. In order to engineer such a gate, we exploit the flux tunability of the SNAIL. In particular, we consider a two-tone modulation of the form

$$
\varphi_{\mathrm{ext}}^{\mathrm{ac}}(t)=\lambda\left[\cos \left(\omega_{r} t\right)+\cos \left(3 \omega_{r} t\right)\right]
$$

where $\lambda \ll 1$ is a small modulation amplitude. This is justified by studying the cubic potential in Eq. (5) in the interaction picture with respect to the free resonator Hamiltonian $\omega_{r} \hat{a}^{\dagger} \hat{a}$. Because of the odd parity of the potential, there are no nonrotating contributions. The terms that are pure in $\hat{a}^{(\dagger)}$ rotate with frequency $\mp 3 \omega_{r}$, while the mixed terms rotate with $\pm \omega_{r}$. Thus, in order to select the full cubic interaction resonantly, the necessity to drive with two frequencies $\omega_{r}$ and $3 \omega_{r}$ arises. It must be pointed out that the drive at $\omega_{r}$ also selects resonantly the linear and the quadratic terms in Eq. (5). However, in Supplemental Material [46], we show that for a realistic choice of parameters the quadratic term is sufficiently suppressed and can thus be neglected. This is not the case for the linear drive. However, its effect can be corrected via a displacement of the resonator field, and, thus, we neglect it in the remainder of this Letter.

Finally, following the above arguments and in the rotating frame, we isolate the desired cubic interaction

$$
\hat{H}_{I}=g_{3}^{\mathrm{ac}}\left(\hat{a}+\hat{a}^{\dagger}\right)^{3},
$$

where the coupling $g_{3}^{\text {ac }}$ depends on the microscopic parameters of the circuit as well as on the modulation amplitude $\lambda$. In principle, one could use classical optimization to determine the circuit parameters that result in Hamiltonian couplings $g_{i}$ which are tailored to specific requirements. However, here we will restrict ourselves to hand-selected circuit parameters based on state-of-the-art realizations of superconducting circuits to demonstrate our claim.

For the chosen parameters, our theory predicts that it is possible to achieve $g_{3}^{\text {ac }} / 2 \pi \approx 0.3 \mathrm{MHz}$ and $\omega_{r} / 2 \pi \approx 4 \mathrm{GHz}$ while the Kerr nonlinearity is tuned to zero [46]. This also guarantees that the drive at $3 \omega_{r}$ is sufficiently detuned from the plasma frequency of the Josephson junctions, which are typically on the order of a few tens of gigahertz [63]. Therefore, we have demonstrated that all of the operations in the set (1) can be implemented with our proposed architecture.

We emphasize that, in principle, more gates are directly accessible through our proposal. While this does not matter for the goal of achieving universality, having at one's disposal customizable high-order gates can lead to substantial advantages when limited to noisy devices with a finite coherence time.

Generation of a cubic phase state.-We now address the generation of a cubic phase state $|\gamma, r\rangle=$ $e^{i \gamma \hat{q}^{3}} e^{(r / 2)\left(\hat{a}^{+2}-\hat{a}^{2}\right)}|0\rangle$, where $r$ is the real squeezing parameter, $\gamma$ the cubicity of the cubic phase gate applied, and $|0\rangle$ the photon vacuum state [26]. Because of the weak coupling to the transmission line, the main dissipation channel corresponds to internal losses. We treat them within a Gorini-Kossakowski-Sudarshan-Lindblad master equation formalism with jump operator $\hat{L}=\sqrt{\kappa} \hat{a}$, where $\kappa$ is the single-photon loss rate. Evolving an initial squeezed state for a time $t_{g}$ with the Hamiltonian (7) results in a cubic phase state with cubicity $\gamma=g_{3}^{\text {ac }} \sqrt{8} t_{g}$, where the factor $\sqrt{8}$ results from the normalization of $\hat{q}$ [46].

At this point, we would like to stress that Hamiltonian (7) is the result of several approximations, more remarkably, the rotating wave approximation. In order to test the validity of the latter approximation, we can apply the above described squeezing and cubic gates by means of the appropriate external drives to the full nonlinear-resonator Hamiltonian Eq. (5) initialized in the vacuum state [46]. In Figs. 2(a) and 2(b), we compare the state resulting from the full Hamiltonian (5) in the presence of losses with the ideal cubic phase state $|\gamma, r\rangle$, corresponding to the lossless evolution under Hamiltonian (7) acting on an ideal squeezed state, respectively. For this example, we consider $\kappa / 2 \pi=50 \mathrm{kHz} \quad(1 / \kappa \approx 3 \mu \mathrm{s})$ which corresponds to a

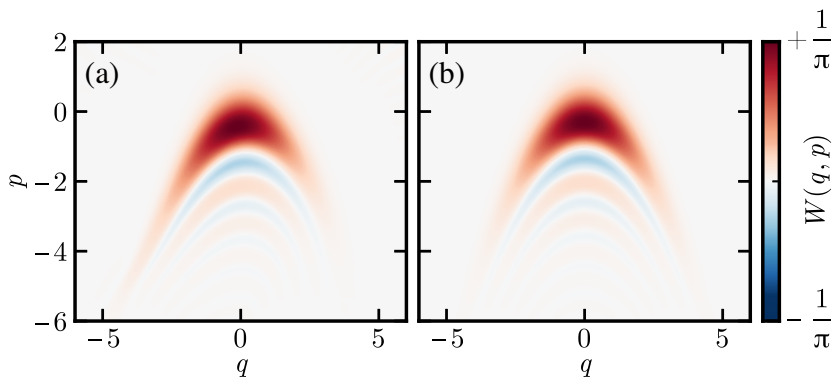

FIG. 2. Wigner distribution for the cubic phase state $|\gamma, r\rangle$. (a) Obtained from a master equation simulation with the Hamiltonian (5) and single-photon loss rate $\kappa / 2 \pi=50 \mathrm{kHz}$ by sequentially applying the squeezing and cubic phase gate to an initial vacuum state $|0\rangle$ (see [46] for details). (b) Ideal cubic phase state with matched cubicity $\gamma \approx 0.1$ and squeezing $r \approx 0.70(\approx 6 \mathrm{~dB})$. 
quality factor $Q$ of the order of $10^{5}$. In addition, we choose evolution times and drive strengths that lead to a squeezing parameter $r \approx 0.7$ [64] and cubicity $\gamma \approx 0.1$ [65]. We obtain a fidelity of $97.2 \%$ to the ideal cubic phase state (numerical simulations are done using QuTiP [66]), which is sufficient to retain its distinguished features, such as the negativities of the Wigner function, as shown in Fig. 2. Note that the fidelity in the absence of losses is increased to $97.4 \%$.

Finally, the generated cubic phase state can be probed, e.g., by quantum state tomography using a dispersively coupled qubit [not shown in Fig. 1(b)] [37].

Conclusions. - In summary, we have proposed a microwave architecture that allows for the implementation of a universal gate set for continuous-variable quantum computation. Our architecture is based on a quarter wavelength transmission line resonator terminated by an array of Josephson junctions in a SNAIL configuration. The tunability of our device allows for engineering customized gates and, in particular, the interaction $\hat{q}^{3}$, corresponding to a cubic phase gate. As an application, we have provided an experimentally realistic protocol for the generation of the cubic phase state, which is a resource state for $\mathrm{CV}$ quantum computation and whose generation has not been experimentally achieved yet despite extensive effort undertaken with quantum optical setups.

On the one hand, our work opens the experimental quest for a cubic phase state with microwave circuits. Our proposal is within reach of current cQED technology in terms of resonator quality factors, that can be as high as $3 \times 10^{5}$ in $3 \mathrm{D}$ architectures [67], and the ability to tune the resonator field much faster than its corresponding lifetime, with pulse synthesis resolution within nanoseconds [68]. On the other hand, the experimental realization of a universal gate set in a continuous-variable architecture would present the community with the question of what relevant quantum algorithms can be run in the near future on such an architecture, possibly with a limited circuit depth, and without fault tolerance. In this sense, our work heralds further research in an area that could be referred to as of "continuous-variable-noisy intermediate scale quantum (CV-NISQ) devices," in resonance with similar investigations recently emerged in the context of qubit-based quantum computation [69]. Indeed, quantum advantage in $\mathrm{CV}$ beyond a specific encoding has been addressed so far only in the context of sampling problems [28,70-75], for the implementation of algorithms for optimization of continuous functions [76], or for numerical integration [77], leaving plenty of room for new applications yet to unveil.

We thank Vitaly Shumeiko for useful discussions. G. F. acknowledges support from the Swedish Research Council (Vetenskapsrådet) Grant QuACVA. F. Q., G. J., S. G., and G. F. acknowledge the financial support from the Knut and Alice Wallenberg Foundation through the Wallenberg Center for Quantum Technology (WACQT). T. H. acknowledges support by the Deutsche Forschungsgemeinschaft via RTG 1995.

[1] S. Haroche, Rev. Mod. Phys. 85, 1083 (2013).

[2] D. J. Wineland, Rev. Mod. Phys. 85, 1103 (2013).

[3] G. Wendin, Rep. Prog. Phys. 80, 106001 (2017).

[4] H. Häffner, C. F. Roos, and R. Blatt, Phys. Rep. 469, 155 (2008).

[5] F. Arute et al., Nature (London) 574, 505 (2019).

[6] N. Ofek, A. Petrenko, R. Heeres, P. Reinhold, Z. Leghtas, B. Vlastakis, Y. Liu, L. Frunzio, S. M. Girvin, L. Jiang et al., Nature (London) 536, 441 (2016).

[7] M. Aspelmeyer, T. J. Kippenberg, and F. Marquardt, Rev. Mod. Phys. 86, 1391 (2014).

[8] S. Lloyd and S. L. Braunstein, Phys. Rev. Lett. 82, 1784 (1999).

[9] V. Parigi, A. Zavatta, M. Kim, and M. Bellini, Science 317, 1890 (2007).

[10] A. Ourjoumtsev, R. Tualle-Brouri, J. Laurat, and P. Grangier, Science 312, 83 (2006).

[11] A. Zavatta, V. Parigi, M. S. Kim, and M. Bellini, New J. Phys. 10, 123006 (2008).

[12] Y.-S. Ra, C. Jacquard, A. Dufour, C. Fabre, and N. Treps, Phys. Rev. X 7, 031012 (2017).

[13] Y.-S. Ra, A. Dufour, M. Walschaers, C. Jacquard, T. Michel, C. Fabre, and N. Treps, Nat. Phys. 16, 144 (2020).

[14] R. de J. León-Montiel, O. S. Magaña-Loaiza, A. Perez-Leija, A. U'ren, K. Busch, A. E. Lita, S. W. Nam, T. Gerrits, and R. P. Mirin, in Frontiers in Optics/Laser Science (Optical Society of America, Washington, DC, 2018), p. LM1B.6.

[15] P. Marek, R. Filip, and A. Furusawa, Phys. Rev. A 84, 053802 (2011).

[16] M. Yukawa, K. Miyata, H. Yonezawa, P. Marek, R. Filip, and A. Furusawa, Phys. Rev. A 88, 053816 (2013).

[17] M. Eaton, R. Nehra, and O. Pfister, New J. Phys. 21, 113034 (2019).

[18] M. Yukawa, K. Miyata, T. Mizuta, H. Yonezawa, P. Marek, R. Filip, and A. Furusawa, Opt. Express 21, 5529 (2013).

[19] M. Lamperti, A. Allevi, M. Bondani, R. Machulka, V. Michálek, O. Haderka, and J. Peřina, in Research in Optical Sciences (Optical Society of America, Washington, DC, 2014), p. QW4B.4.

[20] J. Etesse, M. Bouillard, B. Kanseri, and R. Tualle-Brouri, Phys. Rev. Lett. 114, 193602 (2015).

[21] A. Ourjoumtsev, H. Jeong, R. Tualle-Brouri, and P. Grangier, Nature (London) 448, 784 (2007).

[22] F. Arzani, N. Treps, and G. Ferrini, Phys. Rev. A 95, 052352 (2017).

[23] D. Gottesman, A. Kitaev, and J. Preskill, Phys. Rev. A 64, 012310 (2001).

[24] K. Miyata, H. Ogawa, P. Marek, R. Filip, H. Yonezawa, J.-i. Yoshikawa, and A. Furusawa, Phys. Rev. A 93, 022301 (2016).

[25] R. Yanagimoto, T. Onodera, E. Ng, L. G. Wright, P. L. McMahon, and H. Mabuchi, Phys. Rev. Lett. 124, 240503 (2020).

[26] C. Weedbrook, S. Pirandola, R. García-Patrón, N. J. Cerf, T. C. Ralph, J. H. Shapiro, and S. Lloyd, Rev. Mod. Phys. 84, 621 (2012). 
[27] M. Gu, C. Weedbrook, N. C. Menicucci, T. C. Ralph, and P. van Loock, Phys. Rev. A 79, 062318 (2009).

[28] T. Douce, D. Markham, E. Kashefi, P. van Loock, and G. Ferrini, Phys. Rev. A 99, 012344 (2019).

[29] N. C. Menicucci, Phys. Rev. Lett. 112, 120504 (2014).

[30] K. Fukui, A. Tomita, A. Okamoto, and K. Fujii, Phys. Rev. X 8, 021054 (2018)

[31] C. Vuillot, H. Asasi, Y. Wang, L. P. Pryadko, and B. M. Terhal, Phys. Rev. A 99, 032344 (2019).

[32] K. Noh and C. Chamberland, Phys. Rev. A 101, 012316 (2020).

[33] K. Park, P. Marek, and R. Filip, New J. Phys. 20, 053022 (2018).

[34] O. Houhou, D. W. Moore, S. Bose, and A. Ferraro, arXiv:1809.09733.

[35] M. Brunelli and O. Houhou, Phys. Rev. A 100, 013831 (2019).

[36] X. Gu, A. F. Kockum, A. Miranowicz, Y. xi Liu, and F. Nori, Phys. Rep. 718-719, 1 (2017).

[37] M. Hofheinz, H. Wang, M. Ansmann, R. C. Bialczak, E. Lucero, M. Neeley, A. D. O’Connell, D. Sank, J. Wenner, J. M. Martinis, and A. N. Cleland, Nature (London) 459, 546 (2009).

[38] B. Vlastakis, G. Kirchmair, Z. Leghtas, S. E. Nigg, L. Frunzio, S. Girvin, M. Mirrahimi, M. Devoret, and R. Schoelkopf, Science 342, 607 (2013).

[39] R. Lescanne, M. Villiers, T. Peronnin, A. Sarlette, M. Delbecq, B. Huard, T. Kontos, M. Mirrahimi, and Z. Leghtas, Nat. Phys. 16, 509 (2020).

[40] P. Campagne-Ibarcq, A. Eickbusch, S. Touzard, E. Zalys-Geller, N. E. Frattini, V. V. Sivak, P. Reinhold, S. Puri, S. Shankar, R. J. Schoelkopf et al., arXiv:1907.12487.

[41] N. E. Frattini, U. Vool, S. Shankar, A. Narla, K. M. Sliwa, and M. H. Devoret, Appl. Phys. Lett. 110, 222603 (2017).

[42] N. E. Frattini, V. V. Sivak, A. Lingenfelter, S. Shankar, and M. H. Devoret, Phys. Rev. Applied 10, 054020 (2018).

[43] V. V. Sivak, N. E. Frattini, V. R. Joshi, A. Lingenfelter, S. Shankar, and M. H. Devoret, Phys. Rev. Applied 11, 054060 (2019).

[44] F. Albarelli, M. G. Genoni, M. G. A. Paris, and A. Ferraro, Phys. Rev. A 98, 052350 (2018).

[45] R. Takagi and Q. Zhuang, Phys. Rev. A 97, 062337 (2018).

[46] See Supplemental Material at http://link.aps.org/ supplemental/10.1103/PhysRevLett.125.160501 for details, which includes Refs. [47-50].

[47] M. Leib, F. Deppe, A. Marx, R. Gross, and M. J. Hartmann, New J. Phys. 14, 075024 (2012).

[48] M. Wallquist, V. S. Shumeiko, and G. Wendin, Phys. Rev. B 74, 224506 (2006).

[49] E. Zakka-Bajjani, F. Nguyen, M. Lee, L. R. Vale, R. W. Simmonds, and J. Aumentado, Nat. Phys. 7, 599 (2011).

[50] V. V. Sivak, S. Shankar, G. Liu, J. Aumentado, and M. H. Devoret, Phys. Rev. Applied 13, 024014 (2020).

[51] S. Sefi and P. van Loock, Phys. Rev. Lett. 107, 170501 (2011).

[52] S. Sefi, V. Vaibhav, and P. van Loock, Phys. Rev. A 88, 012303 (2013).

[53] J. M. Arrazola, T. R. Bromley, J. Izaac, C. R. Myers, K. Brádler, and N. Killoran, Quantum Sci. Technol. 4, 024004 (2019).

[54] M. H. Devoret and J. M. Martinis, Quantum Inf. Process. 3, 163 (2004).

[55] W. Wustmann and V. Shumeiko, Phys. Rev. B 87, 184501 (2013).
[56] W. Wustmann and V. Shumeiko, Phys. Rev. Applied 8, 024018 (2017)

[57] I.-M. Svensson, A. Bengtsson, P. Krantz, J. Bylander, V. Shumeiko, and P. Delsing, Phys. Rev. B 96, 174503 (2017).

[58] I.-M. Svensson, A. Bengtsson, J. Bylander, V. Shumeiko, and P. Delsing, Appl. Phys. Lett. 113, 022602 (2018).

[59] C. W. S. Chang, C. Sabín, P. Forn-Díaz, F. Quijandría, A. M. Vadiraj, I. Nsanzineza, G. Johansson, and C. M. Wilson, Phys. Rev. X 10, 011011 (2020).

[60] A. Baust, E. Hoffmann, M. Haeberlein, M. J. Schwarz, P. Eder, J. Goetz, F. Wulschner, E. Xie, L. Zhong, F. Quijandría, B. Peropadre, D. Zueco, J.-J. García Ripoll, E. Solano, K. Fedorov, E. P. Menzel, F. Deppe, A. Marx, and R. Gross, Phys. Rev. B 91, 014515 (2015).

[61] W. Pfaff, C. J. Axline, L. D. Burkhart, U. Vool, P. Reinhold, L. Frunzio, L. Jiang, M. H. Devoret, and R. J. Schoelkopf, Nat. Phys. 13, 882 (2017).

[62] M. C. Collodo, A. Potočnik, S. Gasparinetti, J.-C. Besse, M. Pechal, M. Sameti, M. J. Hartmann, A. Wallraff, and C. Eichler, Phys. Rev. Lett. 122, 183601 (2019).

[63] N. A. Masluk, I. M. Pop, A. Kamal, Z. K. Minev, and M. H. Devoret, Phys. Rev. Lett. 109, 137002 (2012).

[64] We emphasize that for any value of squeezing the distinguished features of the state remain. For example, the negativity of its Wigner function depends on the single effective parameter $\gamma e^{3 r}$ [44].

[65] Having at one's disposal one specific value of cubicity is enough for universality. One reason is that arbitrary cubicity value can be achieved complementing the cubic phase gate with additional squeezing gates. In particular, one value of the cubicity that corresponds to implement the $T$ gate within GKP encoding [23] is achievable with our method.

[66] J. R. Johansson, P. D. Nation, and F. Nori, Comput. Phys. Commun. 184, 1234 (2013).

[67] A. Grimm, N. E. Frattini, S. Puri, S. O. Mundhada, S. Touzard, M. Mirrahimi, S. M. Girvin, S. Shankar, and M. H. Devoret, Nature (London) 584, 205 (2020).

[68] M. Sandberg, C. M. Wilson, F. Persson, T. Bauch, G. Johansson, V. Shumeiko, T. Duty, and P. Delsing, Appl. Phys. Lett. 92, 203501 (2008).

[69] J. Preskill, Quantum 2, 79 (2018).

[70] T. R. Bromley, J. M. Arrazola, S. Jahangiri, J. Izaac, N. Quesada, A. D. Gran, M. Schuld, J. Swinarton, Z. Zabaneh, and N. Killoran, Quantum Sci. Technol. 5, 034010 (2020).

[71] U. Chabaud, T. Douce, D. Markham, P. van Loock, E. Kashefi, and G. Ferrini, Phys. Rev. A 96, 062307 (2017).

[72] T. Douce, D. Markham, E. Kashefi, E. Diamanti, T. Coudreau, P. Milman, P. van Loock, and G. Ferrini, Phys. Rev. Lett. 118, 070503 (2017).

[73] C. S. Hamilton, R. Kruse, L. Sansoni, S. Barkhofen, C. Silberhorn, and I. Jex, Phys. Rev. Lett. 119, 170501 (2017).

[74] L. Chakhmakhchyan and N. J. Cerf, Phys. Rev. A 96, 032326 (2017).

[75] A. P. Lund, S. Rahimi-Keshari, and T. C. Ralph, Phys. Rev. A 96, 022301 (2017).

[76] G. Verdon, J. M. Arrazola, K. Brádler, and N. Killoran, arXiv:1902.00409.

[77] P. Rebentrost, B. Gupt, and T. R. Bromley, arXiv:1809.02579. 